\title{
Dynamic screening of ions passing through solids
}

\author{
D H Jakubassa† \\ Physik-Department, Technische Universität München, 8046 Garching, Germany
}

Received 14 March 1977, in final form 14 July 1977

\begin{abstract}
The screened potential of an ion moving through a free-electron gas is calculated, and the resulting energy shift of bound electronic states of the ion is estimated. We find a decrease of the $2 \mathrm{p}-1 \mathrm{~s}$ transition energy due to screening effects, and compare it with experjmental results for high-velocity $\mathrm{S}$ projectiles in an $\mathrm{Al}$ target.
\end{abstract}

\section{Introduction}

It is well known (Neufeld and Richie 1955, Brandt 1975) that the potential of ions is modified when they pass through solids. The screening is due to the valence electrons of the medium and can be described by means of the dielectric constant $\epsilon(q, \omega)$ of a freeelectron gas (Lindhard 1954). For fast ions with velocity $v$ exceeding the Fermi velocity $v_{\mathrm{F}}$ of the electron gas, Neelavathi et al (1974) and Ritchie et al (1976) derived the screened ion potential by taking an analytical approximation for the dielectric constant. This potential shows oscillations behind the ion which correspond to the excitation of plasmons. Day (1975) used the semiclassical $\epsilon$ but evaluated the potential only along the ion track. Calculating the potential more carefully we find an oscillatory behaviour only in part of the space behind the ion.

Apart from the wake field there is a screening contribution to the potential in the vicinity of the ion which exists for all velocities $v$ and which weakens the Coulomb potential. It leads to a decrease of the binding energy of the electronic states. While for a fast moving ion the deviation from the energy levels of the nuclear Coulomb field tends to zero with increasing $v$, the binding energy is considerably lowered in the case of a slow moving ion.

The relative change of the binding energy is largest for light ions. The ground state of a stationary proton is only very weakly bound (Rogers et al 1970); in fact, a bound state exists only for media with $d_{\mathrm{s}} / a \gtrsim 0.88$ where $d_{\mathrm{s}}$ is the static screening constant and $a$ the Bohr radius (Almbladh et al 1976). We will show that there is no bound state for a slowly moving proton. Whether the bound state which reappears for large $v$ is actually occupied is discussed elsewhere (Cross 1977).

For heavier ions the change in the binding energy can be found by examining the transition energy between electronic states. In a recent experiment (Bell et al 1976a) the $\mathrm{x}$-ray energy of a specific $2 \mathrm{p}$-1s multiplet transition $\left({ }^{1} \mathrm{P}_{1}{ }^{-1} \mathrm{~S}_{0}\right)$ in helium-like sulphur projectiles was measured. A small though distinct line shift was observed depending on whether the transition occurred inside or outside the target.

$\uparrow$ And GSI, Darmstadt, Germany. 
In this paper we first discuss the screened potential $(\$ 2)$, then we estimate the binding energy of the hydrogen ground state in terms of the velocity $v(\S 3)$, and finally we calculate the energy shift of electronic transitions due to dynamical screening in the limit of high velocities $v / v_{\mathrm{F}} \gg 1(\S 4)$ and compare with experiment $(\S 5)$.

\section{Potential of a moving charge}

For weak perturbations, i.e., small ion charges $Z$, the potential generated by a moving ion in the medium can be calculated in linear response theory. The energy of an electron in this potential is given by (Neelavathi et al 1974)

$$
V\left(\rho, z^{\prime}\right)=-2 Z e^{2} / \pi \operatorname{Re}\left\{\int_{0}^{\infty} \mathrm{d} q \int_{0}^{1} \mathrm{~d} x \exp \left(\mathrm{i} q z^{\prime} x\right) J_{0}\left[q \rho \sqrt{ }\left(1-x^{2}\right)\right] / \epsilon(q, \omega)\right\},
$$

where the ion is supposed to move in the $z$ direction with constant velocity $v \ll c$, and $\rho$ and $z^{\prime}=z-v t$ are cylindrical coordinates in the rest system of the ion, $\omega$ is given by $q v=q v x$. The calculation is greatly simplified if one replaces $\epsilon$ by its semiclassical approximation (Lindhard 1954, Day 1975) which agrees with the exact $\epsilon$ for small momenta $q \ll k_{\mathrm{F}}$ or high frequencies $\omega \gg \hbar q^{2} / 2 m$, where $m$ is the electron mass. Since for $v>v_{\mathrm{F}}$ the main contributions to the integral (2.1) come from the region where these conditions are fulfilled, the semiclassical dielectric constant provides a good approximation in this case:

$$
\epsilon(q, q v x)=1-\left(3 \omega_{\mathrm{p}}^{2} / q^{2} v_{\mathrm{F}}^{2}\right) Q_{1}\left(v x / v_{\mathrm{F}}\right)
$$

with $\omega_{\mathrm{p}}$ the plasma frequency and $Q_{1}$ a function independent of $q$ :

$$
Q_{1}(z)=(z / 2) \ln \left|\frac{z+1}{z-1}\right|-1-\mathrm{i} \pi z / 2 \Theta(1-z) .
$$

The imaginary part of $\epsilon$ is nonzero only for $x<v_{\mathrm{F}} / v$ and leads to a contribution to $V$ which is asymmetric with respect to $z^{\prime}$. If $v>v_{\mathrm{F}}$, we split the integral (2.1) into two parts,

$$
V=V_{1}+V_{2} \text {, corresponding to } \int_{0}^{1} \mathrm{~d} x=\int_{0}^{v_{\mathrm{F}} / v} \mathrm{~d} x+\int_{v_{\mathrm{F}} / v}^{1} \mathrm{~d} x
$$

The first part, $V_{1}$, falls off as the inverse cube of the distance for large $\rho, z^{\prime}$, while for small distances it reproduces the Coulomb field $V_{\mathrm{C}}$ plus a positive shift:

$$
V_{1}\left(\rho=0, z^{\prime}=0\right)-V_{\mathrm{C}}=Z e^{2} / d_{\mathrm{s}} \begin{cases}1-\alpha\left(v / v_{\mathrm{F}}\right)^{2} & \text { for } v<v_{\mathrm{F}} \\ \pi /(2 \sqrt{3}) v_{\mathrm{F}} / v & \text { for } v \geqslant v_{\mathrm{F}} .\end{cases}
$$

For low velocities $v<v_{\mathrm{F}}$ there is only a small deviation $(\alpha \approx 0 \cdot 1)$ from the static screening constant $d_{\mathrm{s}}=\left(\sqrt{3} \omega_{\mathrm{p}} / v_{\mathrm{F}}\right)^{-1}$ while for large $v$ the screening tends to zero with $v^{-1}$ (Ritchie et al 1976).

The second part of $V, V_{2}$, exists only in the region $v>v_{\mathrm{F}}$ where $\epsilon^{-1}(q, \omega)$ has poles

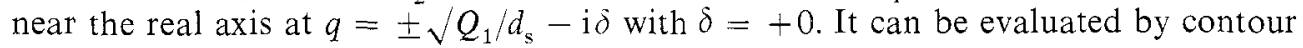
integration and one obtains half of the residue of each pole. Taking into account that the Bessel function $J_{0}(q)$ consists of two parts which behave like $\mathrm{e}^{\mathrm{i} q}$ and $\mathrm{e}^{-\mathrm{i} q}$ respectively, when $q \rightarrow \pm \mathrm{i} \infty$, one obtains in the region $z^{\prime}<-\rho\left[\left(v / v_{\mathrm{F}}\right)^{2}-1\right]^{1 / 2}$. 
$V_{2}=-Z e^{2} / d_{\mathrm{s}} \int_{v_{\mathrm{F}} / v}^{1} \mathrm{~d} x \sin \left(\sqrt{ } Q_{1} z^{\prime} x / d_{\mathrm{s}}\right) J_{0}\left(\sqrt{ } Q_{1} \rho \sqrt{ }\left(1-x^{2}\right) / d_{\mathrm{s}}\right) \sqrt{ } Q_{1}$.

In this region behind the ion, which corresponds to the Cherenkov cone in electrodynamics (Jackson 1975) if one interprets $v_{F}$ as the velocity of light in the medium, $V_{2}$ exhibits damped oscillations. These oscillations are largest on the negative $z^{\prime}$ axis and decrease with growing angle $\theta=\tan ^{-1} \rho /\left|z^{\prime}\right|$, until they disappear for $\rho /\left|z^{\prime}\right| \approx\left(\left(v / v_{\mathrm{F}}\right)^{2}-1\right)^{-1 / 2}$. Figure 1 displays the magnitude of $V_{2}$ at the first minimum as a function of $\theta$. The edge of the cone where the oscillations disappear is smeared out because of damping effects, due to the dispersive $\epsilon(q, \omega)$.

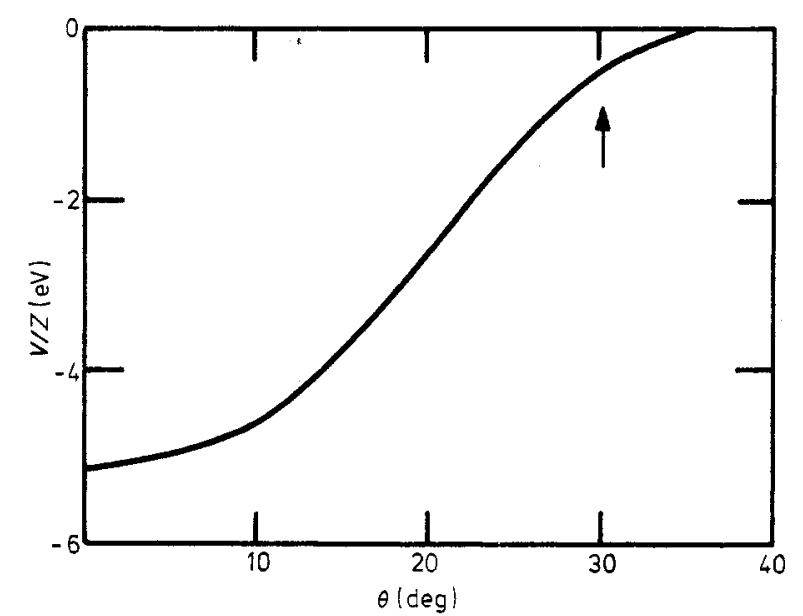

Figure 1. Wake potential $V_{2}$ at its first minimum as a function of the angle between the negative $z^{\prime}$ axis and the ray $z^{\prime} / \rho=$ constant, for $v / v_{\mathrm{F}}=2$ and an $\mathrm{Al}$ target. The arrow denotes the Cherenkov angle.

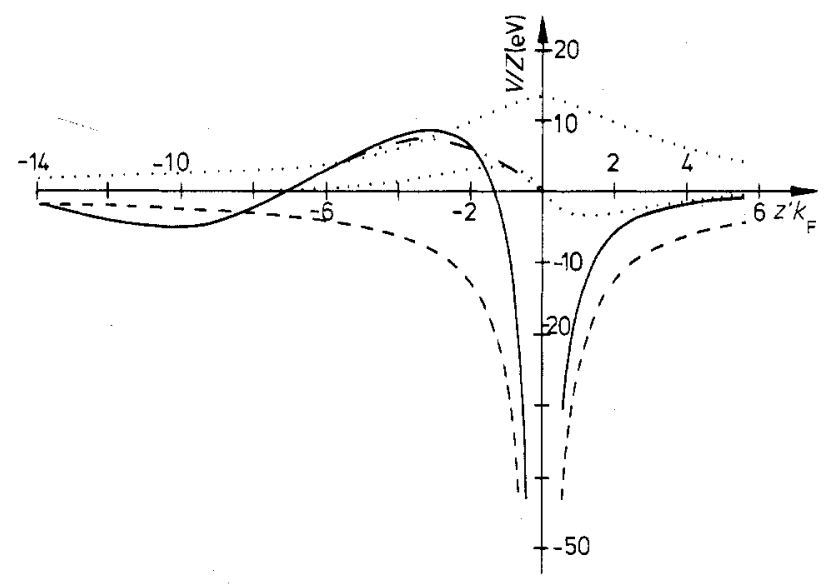

Figure 2. Contributions to the ion potential $V$ in the plane $\rho=0$ for $v / v_{\mathrm{F}}=2$ (Al target). The broken curve is the unscreened Coulomb potential $V_{c}$, the dotted curve is the symmetric and asymmetric part of $V_{1}-V_{C}$, the chain curve is the wake field $V_{2}$ and the full curve gives the total $V$ 
Figure 2 shows the contributions to the screened ion potential in the plane $\rho=0$ as a function of $z^{\prime}$. The potential is strongly asymmetric since the screening electrons of the medium prefer to gather behind the ion. This effect is the more pronounced the higher the ion velocity.

\section{Energy shift of the hydrogen ground state}

The energy levels of an ion passing through a medium are obtained by solving the Schrödinger equation with the potential (2.1). Since we are, however, interested only in the energy shift which is due to the screening we replace the exact energy by its expectation value formed with the unscreened hydrogen wavefunctions. With $V_{\mathrm{sc}}=V-V_{\mathrm{C}}$ the energy shift is given by:

$$
\Delta E_{n} \approx\left\langle\psi_{n}\left(\rho, z^{\prime}\right)\left|V_{\mathrm{sc}}\left(\rho, z^{\prime}\right)\right| \psi_{n}\left(\rho, z^{\prime}\right)\right\rangle .
$$

The main contribution to $\Delta E$ comes from the symmetric part of $V_{1}-V_{C}$ since the expectation value (3.1) is sensitive only to distances close to the ion. Neglecting $V_{2}$ we obtain $\Delta E_{1 \mathrm{~s}}$ from (3.1) and (2.1) by first performing the integration over space:

$$
\Delta E_{1 \mathrm{~s}}=-Z e^{2} / \pi \operatorname{Re} \int_{-\infty}^{\infty} \mathrm{d} q \frac{1}{\left[1+(q a / 2)^{2}\right]^{2}} \int_{0}^{1} \mathrm{~d} x\left(\frac{q^{2} d_{\mathrm{s}}^{2}}{q^{2} d_{\mathrm{s}}^{2}-Q_{1}}-1\right)
$$

with $a=\hbar^{2} /\left(m Z e^{2}\right)$. The $q$ integral can be evaluated by contour integration.

Figure 3 shows the ground state energy of hydrogen passing with velocity $v$ through the electron gas, While for $Z=1$ a bound state exists only for $v / v_{\mathrm{F}} \geq 2$, ions with $Z \geqslant 2$ have bound states for all velocities. For $v \gg v_{F}, E_{1 s}$ approaches the free-ion energy.

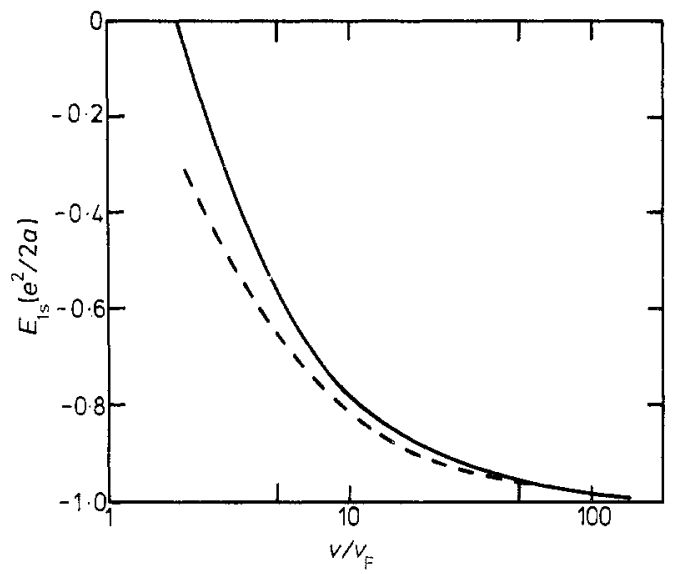

Figure 3. Ground state energy of hydrogen moving with velocity $v$ through an $\mathrm{Al}$ target. The broken curve is obtained by the scaling procedure described in the text.

If $v>v_{\mathrm{F}}$ and $\left(d_{\mathrm{s}} / a\right)\left(v / v_{\mathrm{F}}\right)>1$, the bound state energy can also be obtained by a scaling procedure: one approximates the potential $V$ by the static form $-Z e^{2} / r e^{-r / d}$, valid for small distances, where $d$ is now the 'dynamic' screening constant (Lindhard 1976) $\left(\pi \omega_{\mathrm{p}} / 2 v\right)^{-1}$ from (2.3). The dependence of the energy on the screening constant $d$, obtained by an exact solution of the Schrödinger equation (Rogers et al 1970), can then be transformed into a dependence on $v$. This approximation is also shown in figure 3. 


\section{Energy shift of the atomic transitions}

The energy shift of the atomic levels is again approximated by (3.1). But now we are interested in the difference between the energy levels where the constant potential shift (2.3) cancels, and the wake potential $V_{2}$ can no longer be neglected.

For $Z \gg 1$ we can expand the integrand in (2.1), after having performed the $q$ integration, in terms of $\rho$ and $z^{\prime}$ since $\rho, z^{\prime} \leqslant a$ and $a / d_{\mathrm{s}} \sim 1 / Z$, and then calculate the expectation value with this potential approximation. When integrating over $q$ it is convenient to use the integral representation of $J_{0}$.

The transition energies are modified if there are electrons bound to the ion, since they change the screened potential (2.1). In the case where one $1 \mathrm{~s}$-electron is bound, the additional shift of the transition energy, which arises from the dynamic screening alone, is of the order $1 / Z$ compared to the shift without a bound electron. Both contributions depend quadratically on the inverse screening constant $d^{-1}$.

If $v \gg v_{\mathrm{F}}$, the terms in $V_{\mathrm{sc}}$ involving $\rho$ strongly dominate the terms involving $z^{\prime}$. Neglecting contributions proportional to $z^{\prime} / \rho$ one can approximate $V_{\mathrm{sc}}$ for small distances by $V_{\mathrm{sc}}\left(\rho, z^{\prime}\right) \approx V_{\mathrm{sc}}(\rho, 0)+V_{\mathrm{sc}}\left(0, z^{\prime}\right)-V_{\mathrm{sc}}(0,0)$, and evaluate the transition energy analytically. Considering, for example, the transition from the $2 \mathrm{p}(m= \pm 1)$ state to the 1 s state we find from (2.1):

$$
\Delta E_{2 \mathrm{p}-1 \mathrm{~s}}^{( \pm 1)} \approx-\frac{Z e^{2} a}{16 d_{\mathrm{c}}^{2}}\left(v_{\mathrm{F}} / v\right)^{2}\left(\frac{33}{4} v / v_{\mathrm{F}}-3 \ln v / v_{\mathrm{F}}-3 \ln 2+\frac{41}{4}\right)
$$

which deviates less than $10 \%$ from the numerical value. The energy shift decreases with $v^{-1}$ for large velocities, and is in zero order, independent of $Z$ in the region where the linear response theory is valid.

The dependence of $\Delta E_{2 \mathrm{p}-1 \mathrm{~s}}^{( \pm 1)}$ on the density $n$ of the electron gas is shown in figure 4. The increase of (4.1) with $n$ is nearly independent of the velocity $v$.

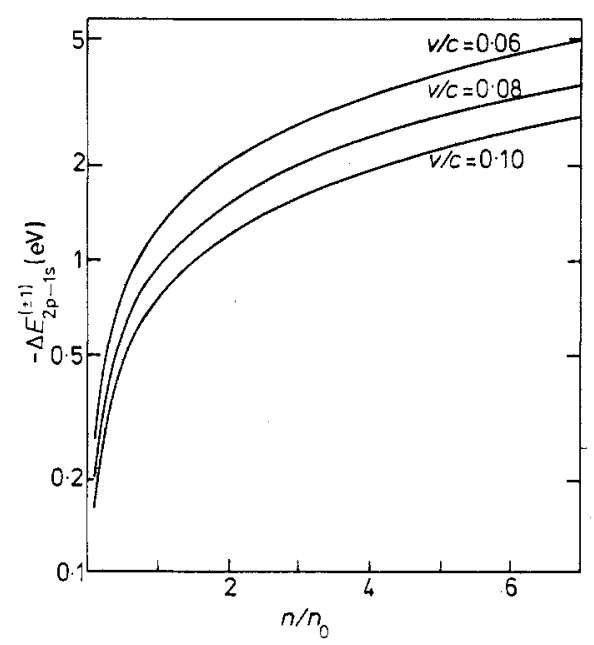

Figure 4. Shift of the transition energy between the $2 \mathrm{p}$ and $\mathrm{ks}$ state as a function of the density of the medium for various ion velocities $\left.t \cdot n_{0}=10^{-16} \mathrm{fm}^{-3}\right]$. 


\section{Comparison with experiment and discussion}

In a recent experiment (Bell et al 1976a) the $\mathrm{x}$-ray energy of the $2 \mathrm{p}-1 \mathrm{~s}$ transition in helium-like sulphur projectiles with $v / c=0 \cdot 08$ has been measured as a function of the thickness of an $\mathrm{Al}$ target $\left(v / v_{\mathrm{F}}=11 \cdot 8\right)$. The transition energy depends on the ratio of decays inside and outside the target, i.e., on the ratio of the lifetime $\lambda^{-1} \approx 1.5 \times 10^{-14} \mathrm{~s}$ of the $\mathrm{K}$ hole to the time of flight $t_{x}=x / v$ through the target of thickness $x$. For a qualitative description of the target thickness dependence of the energy we assume a constant number $I_{0}$ of $\mathrm{K}$ holes inside the target and an exponential decay $I=I_{0} \mathrm{e}^{-\lambda t}$ outside the target. The $\mathrm{X}$-ray yield from decays inside the target is then given by $I_{0} t_{x} / \tau_{\mathrm{R}}$, and for decays from outside one obtains $I_{0} /\left(\lambda \tau_{\mathrm{R}}\right)\left(\right.$ Bell et al 1976b), where $\tau_{\mathrm{R}}$ is the radiative lifetime of the $2 p$ state. Normalising by the total yield we find for the mean transition energy:

$$
\bar{E}_{2 \mathrm{p}-1 \mathrm{~s}}=E_{2 \mathrm{p}-1 \mathrm{~s}}+\Delta E_{2 \mathrm{p}-1 \mathrm{~s}} \frac{\lambda t_{x}}{1+\lambda t_{x}}
$$

where $E_{2 p-1 \mathrm{~s}}$ is the transition energy for decays outside the target. Figure 5 shows the target thickness dependence of the energy shift $\bar{E}_{2 \mathrm{p}-1 \mathrm{~s}}-E_{2 \mathrm{p}-1 \mathrm{~s}}$ of the ${ }^{1} P_{1}-{ }^{1} S_{0}$ transition. The metastable ${ }^{3} \mathrm{P}_{1}-{ }^{1} \mathrm{~S}_{0}$ transition was taken as a reference line since it is independent of $x$. The theory (5.1) is normalised to experiment at the smallest target thickness since $\Delta E_{2 \mathrm{p}-1 \mathrm{~s}}$ only yields the energy difference of decays for $x=0$ and $x=\infty$. We calculated $\Delta E_{2 \mathrm{p}-1 \mathrm{~s}}$ and found $-1.17 \mathrm{eV}$ for the $2 \mathrm{p}(m= \pm 1)$ initial state and $-1.06 \mathrm{eV}$ for $m=0$. For the evaluation of (5.1) we averaged over $m$ and obtained $\Delta E_{2 \mathrm{p}-1 \mathrm{~s}}=-1 \cdot 13 \mathrm{eV}$. The experimental energy shift is thus well reproduced.

To summarise, dynamical screening of a moving ion influences all processes which are sensitive to the nuclear charge. At small distances from the ion, compared to the static screening length, we have approximately static screening for $v<v_{\mathrm{F}}$ whereas for large velocities $v \gg v_{\mathrm{F}}$ the screening effects diminish with $v^{-1}$. It leads to a decrease of the binding energies of the electronic states of the ion, as well as to a lowering of the electronic

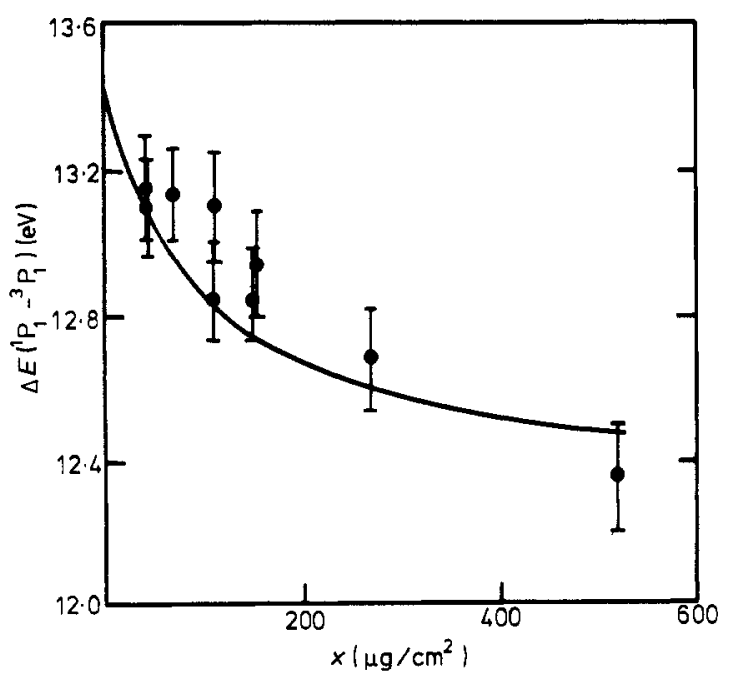

Figure 5. Energy difference between the ${ }^{1} \mathrm{P}_{1}-{ }^{1} \mathrm{~S}_{0}$ and ${ }^{3} \mathrm{P}_{1}-{ }^{1} \mathrm{~S}_{0}$ transition of $\mathrm{S}$ in $\mathrm{Al}$ at $v / \mathrm{c}=0.08$ as a function of the target thickness. The data points are taken from Bell et al (1976a). 
transition energies in agreement with experiment. At large distances behind the ion the potential shows strong oscillations inside the Cherenkov cone for $v>v_{\mathrm{F}}$ which leads to speculations about wake-riding charged particles in dense media (Neelavathi et al 1974). For slow ions with high nuclear charges one has to treat the dynamical screening within a nonlinear theory, but this will not change qualitatively the results mentioned above.

\section{Acknowledgments}

I would like to thank Dr M Kleber and Professor $H$ Schmidt for interesting and stimulating discussions.

\section{References}

Almbladh C O, v Barth U, Popovic Z D and Stott M J 1976 Phys. Rev. B14 2250-4

Bell F, Betz H D, Panke H and Stehling W 1976a J. Phys. B. Atom. Molec. Phys. 9 L443-6

Bell F, Betz H D, Panke H, Stehling W and Spindler E 1976b J. Phys, B. Atom. Molec. Phys. $93017-22$

Brandt W 1975 Atomic Collisions in Solids vol 1, eds Datz S, Appleton B R and Moak C D (New York: Plenum) pp 261-88

Cross M C 1977 Phys. Rev. B15 602-7

Day M H 1975 Phys. Rev. B12 514-6

Jackson J D 1975 Classical Electrodynamics 2nd ed (New York: Wiley) pp 638-41

Lindhard J 1954 Mat. Fys. Medd. Dan. Vid. Selsk. 28 no 8

- $1976 \mathrm{Nucl}$. Instrum. Methods 132 1-5

Neelavathi V N, Ritchie R H and Brandt W 1974 Phys. Rev. Lett. 33 302-5

Neufeld J and Ritchie R H 1955 Phys. Rev. 98 1632-42

Ritchie R H, Brandt W and Echenique P M 1976 Phys. Rev. B $144808-12$

Rogers F J, Graboske H C and Harwood D J 1970 Phys. Rev A1 1577-86 